FOREWORD to Vol. 10

\title{
Special Cluster in Conjunction with IEICE General Conference 2021
}

Since 2019, the editorial committee of ComEX has issued the special cluster related to the IEICE General Conference, which is annually held in March; there we have more than 1000 presentations with one- or two-page manuscripts for each. Since most of them are written in Japanese, this cluster is planned to open the technologies shown there to the world. Thanks to the authors and readers, ComEX's annual submissions and published letters are increasing; we think that these clusters enjoy good reputations and push the numbers up. Finally, I would like to thank all the authors, reviewers, and the editorial committee members, for their contributions and great efforts to make those special clusters successful.

\section{Guest Editor-in-Chief:}

Makoto Taromaru (Fukuoka Univ.)

\section{Special Cluster Editorial Committee Members}

Guest Editors: Noriaki Kamiyama (Ristumeikan Univ.), Daisuke Umehara (Kyoto Inst. of Tech.)

Guest Associate Editors: Yuyuan Chang (Tokyo Inst. of Tech.), Chiao-En Chen (National Chung Cheng Univ.), Young-June Choi (Ajou Univ.), Chun-I Fan (National Sun Yat-sen Univ.), Mariusz Głąbowski (Poznan Univ. of Tech.), Bo Gu (Sun Yat-sen Univ.), Guan Gui (NUPT), Yusuke Hirota (NICT), Ezra Ip (NEC Labs. America), Tomohiro Ishihara (Univ. of Tokyo), Hiroshi Kubo (Ritsumeikan Univ.), Ayumu Kubota (KDDI R\&D), Shigeaki Kuzuoka (Wakayama Univ.), Zhetao Li (Xiangtan Univ.), Richard T.B. Ma (National Univ. of Singapore), Tzyh-Ghuang Ma (NTUST), Hiroaki Morino (Shibaura Inst. of Tech.), Hoang Nam Nguyen (Vietnam National Univ., Hanoi), Eisuke Nishiyama (Saga Univ.), Wakaha Ogata (Tokyo Inst. of Tech.), Masakatsu Ogawa (Sophia Univ.), Chuwong Phongcharoenpanich (KMIT Ladkrabang), Nordin Ramli (MIMOS Berhad), Kentaro Saito (Tokyo Denki Univ.), Takatoshi Sugiyama (Kogakuin Univ.), Hidenori Takahashi (KDDI R\&D), Shuto Yamamoto (NTT), Hui Zhang (Nankai Univ.), Miao Zhang (Xiamen Univ.) 